ISSN 1810-0198. Вестник Тамбовского университета. Серия: естественные и технические науки

Том 23, № 124

2018

DOI: $10.20310 / 1810-0198-2018-23-124-595-604$

\title{
NEW SUFFICIENT CONDITIONS IN THE GENERALIZED SPECTRUM APPROACH TO DEAL WITH SPECTRAL POLLUTION
}

\author{
\& A. Khellaf \\ Université 8 Mai 1945, Guelma \\ B.P. 401 Guelma Algérie \\ E-mail: amarlasix@gmail.com
}

\begin{abstract}
In this work, we propose new sufficient conditions to solve the spectral pollution problem by using the generalized spectrum method. We give the theoretical foundation of the generalized spectral approach, as well as illustrate its effectiveness by numerical results.

Keywords: generalized spectrum, Schrödinger operator, eigenvalue approximation
\end{abstract}

\section{Introduction}

Spectral approximation for differential operators takes place in different applications in conjunction with the study of the mathematical modeling, as the case of Schrödinger operator in the quantum physics. Numerical discretization of these problems leads to spurious results, a phenomenon known as spectral pollution (see e.g. [1-5]). In this work, we establish new sufficient conditions to deal with the spectral pollution by using the generalized spectrum method. This method was introduced in [6], and recently was developed in [7].

Let $T$ and $S$ be two bounded operators defined on Banach space $X$, we define the generalized resolvent set by

$$
r e(T, S)=\} z / \mathbb{C}:\left(\begin{array}{ll}
T & z S
\end{array}\right) \text { is bijective } \mid .
$$

The complementary of the generalized resolvent set is the generalized spectrum set, denoted by $s p(T, S)$. We say that $\lambda$ is a generalized eigenvalue of $(T, S)$ if there exists $u / X \quad\} 0 \mid$ such that $T u=\lambda S u$ (see [8]).

In [6], it is shown that the Schrödinger operator, say $A$, has a decomposition into two bounded operators, say $T$ and $S$, that allows to express its spectrum in terms of generalized spectrum, i.e.

$$
s p(A)=s p(T, S) .
$$

Through numerical approximation of the bounded operators $T$ and $S$ by sequences of bounded operators $\left(T_{n}\right)_{n\{\mathbb{N}}$ and $\left(S_{n}\right)_{n\{\mathbb{N}}$, we can prove that $\lim _{n \prime} s p\left(T_{n}, S_{n}\right)=s p(T, S)$, 
where the limit is defined to satisfy the property U: if $T_{n} \Rightarrow T, S_{n} \Rightarrow S, \lambda_{n} / \operatorname{sp}\left(T_{n}, S_{n}\right)$ and $\lambda_{n} \Rightarrow \lambda$, then $\lambda / s p(T, S)$.

This propriety $\mathrm{U}$ is a natural extension of the classical case $S=I$ (see [9]).

In [6], the author showed that the Propriety $U$ is valid under the norm convergence of $\left(T_{n}\right)_{n\{\mathbb{N}}$ and $\left(S_{n}\right)_{n\{\mathbb{N}}$ to $T$ and $S$. In this work, we show that under the collectively compact convergence of $\left(T_{n}\right)_{n\{\mathbb{N}}$ and $\left(S_{n}\right)_{n\{\mathbb{N}}$ to $T$ and $S$, the propriety $\mathrm{U}$ also takes place. Finally, our numerical application (see [7]) shows the coherence and effectiveness of the generalized spectrum method in comparison with other methods.

\section{Generalized spectral approximation under collectively compact convergence}

In this section, we prove that the propriety $U$ can be obtained under the collectively compact convergence. Let $X$ be a Banach space, we denote by $B L(X)$ the space of bounded linear operators acting on $X$. Let $T$ and $S$ be two operators in $B L(X)$. We assume that there exist $\left(T_{n}\right)_{n\{\mathbb{N}}$ and $\left(S_{n}\right)_{n\{\mathbb{N}}$ in $B L(X)$ such that

(B1) $T_{n} \stackrel{c c}{\Rightarrow} T$,

(B2) $S_{n} \stackrel{c c}{\Rightarrow} S$

where $S_{n} \stackrel{c c}{\Rightarrow} S$ stands for the collectively compact convergence, i.e. if the set

$$
\left.\bigcup_{n \rightarrow n_{0}}\right\} S_{n} x \quad S x: x / X, \backslash x \backslash_{X}=1 \mid
$$

is relatively compact in $X$ and for all $x / X, S_{n} x \Rightarrow S x$ pointwisely.

In what follows, the pointwise convergence will be denoted by $\stackrel{p}{\Rightarrow} \bigcirc$

In this section, we state a set of lemmas which will be needed in the proofs of our main theorems.

Lemma 1.1. If $T_{n} \stackrel{p}{\Rightarrow} T$ and $S_{n} \stackrel{c c}{\Rightarrow} S$, then for any bounded operator $H$ in $B L(X)$,

$$
\backslash\left(\begin{array}{ll}
T_{n} & T) H\left(S_{n} \quad S\right) \backslash \Rightarrow 0 .
\end{array}\right.
$$

$\mathrm{P}$ r o o f. Since $T_{n} \stackrel{p}{\Rightarrow} T$, and the set

$$
\left.H\left(\bigcup_{n \rightarrow n_{0}}\right\} S x \quad S_{n} x: \backslash x \backslash=1 \mid\right),
$$

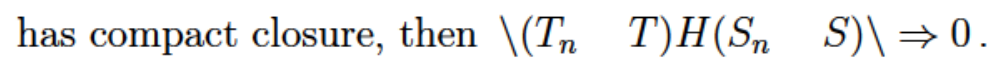

Lemma 1.2. Let $T, \widetilde{T}, S, \widetilde{S} / B L(X)$, and let $z / \operatorname{re}(T, S)$ be such that

$$
\left.\backslash\left[\left(\begin{array}{llll}
(T & \widetilde{T}
\end{array}\right) \quad z\left(\begin{array}{ll}
S & \widetilde{S}
\end{array}\right)\right)\left(\begin{array}{ll}
T & z S
\end{array}\right)^{1}\right]^{2} \backslash<1 .
$$

Then $z / \operatorname{re}(\widetilde{T}, \widetilde{S})$, and

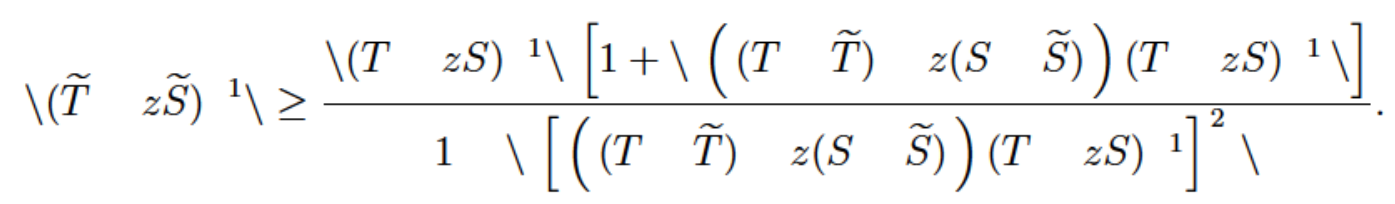


P r o o f. We denote $\widetilde{E}=\left(\begin{array}{lll}T & \widetilde{T}\end{array}\right)\left(\begin{array}{ll}T & z S\end{array}\right)^{1}, \widetilde{F}=\left(\begin{array}{lll}S & \widetilde{S}\end{array}\right)\left(\begin{array}{ll}T & z S\end{array}\right)^{1}$, then

$$
\left.\widetilde{T} \quad z \widetilde{S}=\left[\begin{array}{lll}
I & (\widetilde{E} & z \widetilde{F}
\end{array}\right)\right]\left(\begin{array}{ll}
T & z S
\end{array}\right) .
$$

So, by using the second Neumann expansion (see, [9]), we obtain that

$$
\begin{aligned}
& (\widetilde{T} \quad z \widetilde{S})^{1}=\left(\begin{array}{ll}
T & z S
\end{array}\right)^{1} \sum_{k=0}^{\in}(\widetilde{E} \quad z \widetilde{F})^{2 k} \\
& +(T \quad z S)^{1} \sum_{k=0}^{\epsilon}(\widetilde{E} \quad z \widetilde{F})^{2 k+1} \\
& \left.=\left(\begin{array}{ll}
T & z S
\end{array}\right)^{1}\left[\begin{array}{ll}
I+(\widetilde{E} & z \widetilde{F}
\end{array}\right)\right] \sum_{k=0}^{\epsilon}\left[\begin{array}{ll}
\widetilde{E} & z \widetilde{F})^{2}
\end{array}\right]^{k} \text {. } \\
& \backslash(\widetilde{T} \quad z \widetilde{S})^{1} \backslash \geq \frac{\backslash\left(\begin{array}{lll}
T & z S
\end{array}\right)^{1} \backslash\left(\begin{array}{cc}
1+\backslash \widetilde{E} & z \widetilde{F} \backslash)
\end{array}\right.}{1 \quad \backslash(\widetilde{E} \quad z \widetilde{F})^{2} \backslash}
\end{aligned}
$$

P r o p o s i t i o n 1.1. If (B1) and (B2) are obtained, then for each $z / \operatorname{re}(T, S), z$ belongs to $\operatorname{re}\left(T_{n}, S_{n}\right)$ for big enough $n$.

P r o o f. Let $z / \operatorname{re}(T, S)$, for big enough $n$ we consider

$$
T_{n} \quad z S_{n}=\left[\begin{array}{lll}
I & \left(\widetilde{E}_{n}\right. & z \widetilde{F}_{n}
\end{array}\right]\left(\begin{array}{ll}
T & z S
\end{array}\right),
$$

where $\widetilde{E}_{n}=\left(\begin{array}{lll}T & T_{n}\end{array}\right)\left(\begin{array}{ll}T & z S\end{array}\right)^{1}$ and $\widetilde{F}_{n}=\left(\begin{array}{ll}S & S_{n}\end{array}\right)\left(\begin{array}{ll}T & z S\end{array}\right)^{1}$. Firstly, we have

$$
\left(\widetilde{E}_{n} \quad z \widetilde{F}_{n}\right)^{2}=\left(\widetilde{E}_{n}\right)^{2}+\left(z \widetilde{F}_{n}\right)^{2} \quad z \widetilde{E}_{n} \widetilde{F}_{n} \quad z \widetilde{F}_{n} \widetilde{E}_{n}
$$

So, according to lemma 1.1, we find $\backslash\left(\widetilde{E}_{n} \quad z \widetilde{F}_{n}\right)^{2} \backslash \Rightarrow 0$. Thus, by applying lemma 1.2, we obtain that $z / \operatorname{re}\left(T_{n}, S_{n}\right)$ for big enough $n$.

The following theorem shows that the property $U$ is valid under the collectively compact convergence.

Theorem 1.1. Under (B1) and (B2), if for each $n$ big enough, $\lambda_{n} / \operatorname{sp}\left(T_{n}, S_{n}\right)$ and $\lambda_{n} \Rightarrow \lambda$, then $\lambda / \operatorname{sp}(T, S)$.

P r o of. Assume that $\lambda \forall s p(T, S)$, knowing that $s p(T, S)$ is closed (see e.g. [6]), there exists $r>0$ such that the ball $B(\lambda, r)$ is contained in $r e(T, S)$. Hence according to proposition 1.1, $B(\lambda, r)$ is contained also in $r e\left(T_{n}, S_{n}\right)$ for $n$ big enough. On the other hand, we have $\lambda_{n} \Rightarrow \lambda$. Thus there exists $n_{0}$ such that for any $n \subset n_{0}, \lambda_{n} / B(\lambda, r) \rightarrow \operatorname{re}\left(T_{n}, S_{n}\right)$ which forms the contradiction. 


\section{Numerical application}

As an example, for which the numerical results are available by other approaches, we consider the following problem from [10], which is also studied in [1].

We consider the unbounded operator $A$ defined in $L^{2}(0,+\in)$ by the differential equation

$$
u^{\infty}+x^{2} u=0, \quad u(0)=0 .
$$

This is the harmonic oscillator problem with domain

$$
D(A)=H^{2}(0, \in) \bigcap\left\{u / L^{2}(0, \in): \quad \int_{0}^{\epsilon} x^{2}\|u\|^{2} d x<+\epsilon\right\} .
$$

First, according to the theory of pseudo spectrum for self-adjoint operators (see $[6,7,11]$ ) we can find

$$
s p(A)=\bigcup_{a>0} s p\left(A_{a}\right)
$$

where $A_{a}$ is the Schrödinger operator which has the same formula as $A$ in $L^{2}(0, a)$, but with the Dirichlet condition at the point $a$. The domain of $A_{a}$ is given by

$$
D\left(A_{a}\right)=H^{2}(0, a) \bigcap H_{0}^{1}(0, a) .
$$

Let $a>0$, we denote by $L_{a}$ the Laplacian operator defined on $L^{2}(0, a)$ by

$$
L_{a} u=u^{\infty}, D(L)=H^{2}(0, a) \bigcap H_{0}^{1}(0, a) .
$$

$\mathrm{Pr}$ o p o s ition 2.2. $L_{a}$ is invertible and its inverse is the bounded operator $S_{a}$ defined by

$$
S_{a} u(x)=\int_{0}^{a} G_{\} 0, a}(x, y) u(y) d y, \quad u / L^{2}(0, a)
$$

where

$$
G_{\} 0, a}(x, y)= \begin{cases}\frac{x(a y)}{(a x)} & 0 \geq x \geq y \geq a \\ \frac{y(a x)}{a} & 0 \geq y \geq x \geq a .\end{cases}
$$

P r o o f. See [12].

Let $T_{a}$ be the bounded operator defined on $L^{2}(0, a)$ to itself by

$$
T_{a} u(x)=u(x)+\int_{0}^{a} G_{\}_{0, a}}(x, y) y^{2} u(y) d y, \quad\{x /[0, a] .
$$

Theorem 2.2. $s p(A)=\bigcup_{a>0} s p\left(T_{a}, S_{a}\right)$, 
$\mathrm{P}$ r o o f. According to equality (2.1), we only need to show that $s p\left(A_{a}\right)=s p\left(T_{a}, S_{a}\right)$ for $a>0$. Let $\lambda$ be an eigenvalue of $A_{a}$ with eigenvector $\left.u / D\left(A_{a}\right) \quad\right\} 0 \mid$, by applying $S_{a}$ to $A_{a} u=\lambda u$ we get

$$
T_{a} u=\lambda S_{a} u,
$$

which implies that $\lambda$ is a generalized eigenvalue of the couple $\left(T_{a}, S_{a}\right)$ with eigenvector $\left.u / L^{2}(0, a) \quad\right\} 0 \mid$. Inversely, let $\lambda$ be a generalized eigenvalue of the couple $\left(T_{a}, S_{a}\right)$ with eigenvector $\left.u / L^{2}(0, a) \quad\right\} 0 \mid$, i.e. $T_{a} u=\lambda S_{a} u$, so

$$
u=\lambda S_{a} u \quad S_{a}(v u) \infty u=S_{a}(\lambda u \quad v u),
$$

where $v(x)=x^{2}$. Since $\lambda u \quad v u / L^{2}(0, a)$, we find $u / D\left(L_{a}\right)=D\left(A_{a}\right)$, then

$$
u+S_{a}(v u)=\lambda S_{a} u \infty L_{a} u+v u=\lambda u
$$

Now, we use numerical methods to approach the operators $T_{a}$ and $S_{a}$. We begin by the Nyström method then the Sloan method and the Kantorovich method.

1. Nyström method: We define a subdivision of $[0, a]$ for $n \subset 2$ by

$$
h_{n}=\frac{a}{n \quad 1}, x_{i}=\left(\begin{array}{ll}
i & 1
\end{array}\right) h_{n}, 1 \geq i \geq n
$$

Let $T_{a, n}$ and $S_{a, n}$ be approximation of $T_{a}$ and $S_{a}$ respectively, according to the Nyström method (see [13]),

$$
\begin{aligned}
& T_{a, n} u_{n}(x)=u_{n}(x)+\sum_{i=1}^{n} w_{i} G_{\} 0, a}\left(x, x_{i}\right) x_{i}^{2} u_{n}\left(y_{i}\right), \\
& S_{a, n} u_{n}(x)=\sum_{i=1}^{n} w_{i} G_{\} 0, a}\left(x, x_{i}\right) u_{n}\left(x_{i}\right)
\end{aligned}
$$

where $\}\left.w_{i}\right|_{i=1} ^{n}$ are real weights such that $\sup _{n \rightarrow 2} \sum_{i=1}^{n}|| w_{i} \|<\epsilon$.

Then, we get the matrix generalized eigenvalue problem, $A=\lambda_{n} B$ where

$$
A(i, j)=I(i, j)+w_{i} G_{\}_{0, a}}\left(x_{j}, x_{i}\right) x_{i}^{2}, \quad B(i, j)=w_{i} G_{\}_{0, a}}\left(x_{j}, x_{i}\right),
$$

$I_{n \geq n}$ represents the identity matrix. Finally, we use the function "eig" in Matlab to calculate the generalized eigenvalue of the couple $(A, B)$.

Note that in this case of using the Nyström method, the collectively compact convergence takes place (see [13]). 
2. Sloan method: By using the previous subdivision of $[0, a]$, we define the approximate operators $\widetilde{T}_{a, n}$ and $\widetilde{S}_{a, n}$ of $T_{a}$ and $S_{a}$ respectively, by using the Sloan method (see [9]), i.e. for all $x /[0, a]$,

$$
\begin{aligned}
& \widetilde{T}_{a, n} u_{n}(x)=\sum_{i=1}^{n} u_{n}\left(x_{i}\right) e_{i}(x)+\sum_{i=1}^{n} w_{1, i}(x) u_{n}\left(x_{i}\right), \\
& \widetilde{S}_{a, n} u_{n}(x)=\sum_{i=1}^{n} w_{2, i}(x) u_{n}\left(x_{i}\right)
\end{aligned}
$$

where

$$
w_{1, i}(x)=\int_{0}^{a} G_{\} 0, a}(x, y) y^{2} e_{i}(y) d y, w_{2, i}(x)=\int_{0}^{a} G_{\} 0, a}(x, y) e_{i}(y) d y, 1 \geq i \geq n,
$$

and for $2 \geq i \geq n \quad 1$,

$$
\begin{aligned}
& e_{i}(x)=\left\{\begin{array}{cc}
1 \frac{\left\|x \quad x_{i}\right\|}{h_{n}}, \quad x_{i} \geq x \geq x_{i+1} \\
0, \quad \text { otherwise. }
\end{array}\right. \\
& e_{1}(x)=\left\{\begin{array}{cc}
\frac{x_{2} x}{h_{n},} & x_{1} \geq x \geq x_{2} \\
0, & \text { otherwise. }
\end{array}\right. \\
& e_{n}(x)=\left\{\begin{array}{cc}
\frac{x \quad x_{n} 1}{h_{n},} & x_{n} 1 \geq x \geq x_{n} \\
0, & \text { otherwise. }
\end{array}\right.
\end{aligned}
$$

Then, we get the matrix generalized eigenvalue problem $\widetilde{A}=\lambda_{n} \widetilde{B}$, where

$$
\widetilde{A}(i, j)=I(i, j)+w_{1, i}\left(x_{j}\right), \widetilde{B}(i, j)=w_{2, i}\left(x_{j}\right) .
$$

Finally, we use the function "eig" in Matlab to calculate the generalized eigenvalue of the couple $(\widetilde{A}, \widetilde{B})$.

Note that on the case of the Sloan projection method, the collectively compact convergence also takes place (see [9]).

3. Kantorovich method: By using again the previous subdivision of $[0, a]$, we apply the Kantorovich projection method (see [9]), we get for all $x /[0, a]$

$$
u_{n}(x)+\sum_{i=1}^{n}\left(\int_{0}^{a} G_{\} 0, a}\left(x_{i}, y\right) y^{2} u_{n}(y) d y\right) e_{i}(x)=\lambda_{n} \sum_{i=1}^{n}\left(\int_{0}^{a} G_{\} 0, a}\left(x_{i}, y\right) u_{n}(y) d y\right) e_{i}(x) .
$$

Multiplying first by $G_{\} 0, a}\left(x_{j}, x\right) x^{2}$ then by $G_{\} 0, a}\left(x_{j}, x\right)$ and integrating over $[0, a]$ the equation (2.2), this leads to the matrix generalized eigenvalue problem

$$
\left[\begin{array}{cc}
\widetilde{A}+I_{n \geq n} & O_{n \geq n} \\
\widetilde{B} & I_{n \geq n}
\end{array}\right]\left[\begin{array}{l}
\beta_{1} \\
\beta_{2}
\end{array}\right]=\lambda_{2 n}\left[\begin{array}{cc}
O_{n \geq n} & \widetilde{A} \\
O_{n \geq n} & \widetilde{B}
\end{array}\right]\left[\begin{array}{l}
\beta_{1} \\
\beta_{2}
\end{array}\right],
$$


Table 1. The numerical results for $\mathrm{a}=5$

\begin{tabular}{cccc}
\hline Nystrom & Sloan & Kantorovich & results of [10] \\
\hline 2.9998027 & 3.0001972 & 3.0001972 & 2.9621125 \\
6.9990159 & 7.0009887 & 7.0009887 & 6.8083144 \\
10.9977898 & 11.0026039 & 11.0026039 & 10.5272610 \\
15.0013776 & 15.0103317 & 15.0103317 & 14.1401140 \\
19.0656824 & 19.0806050 & 19.0806050 & 17.8348945 \\
\hline
\end{tabular}

where

$$
\beta_{1}(j)=\int_{0}^{a} G_{\}_{0, a}}\left(x_{j}, y\right) y^{2} u_{n}(y) d y, \beta_{2}(j)=\int_{0}^{a} G_{\}_{0, a}}\left(x_{j}, y\right) u_{n}(y) d y, 1 \geq j \geq n,
$$

and $(\widetilde{A}, \widetilde{B})$ are the same matrices presented in the Sloan method. Finally, we use again the function "eig" in Matlab to calculate the generalized eigenvalue of

$$
\left(\left[\begin{array}{cc}
\widetilde{A}+I_{n \geq n} & O_{n \geq n} \\
\widetilde{B} & I_{n \geq n}
\end{array}\right],\left[\begin{array}{cc}
O_{n \geq n} & \widetilde{A} \\
O_{n \geq n} & \widetilde{B}
\end{array}\right]\right)
$$

In this case of the Kantorovich projection method, the norm convergence takes place (see [9]).

We fix $n=200$ to approach the eigenvalues in our example by using the three numerical methods, we compare our results with those in [10]. Table (3.) shows the numerical results.

\section{Conclusion}

Our study shows the efficiency of the generalized spectrum method, from both theoretical and numerical points of view. This technique appears to be a computationally attractive tool for resolving the spectral pollution. We resolved it by treating the analytical question, to find the bounded operators $T$ and $S$ representing the spectrum proprieties of the Schrödinger operator in the theory of generalized spectrum.

As perspective, we will try to answer this question in more complicated case, for two dimensions and then for three dimensions, where the geometry of the domain and the boundary conditions, will form the main part of the problem. We will also try to generalize this method to other unbounded operators.

\section{REFERENCES}

1. Aslanyan A., Davies E.B. Spectral instability for some Schrodinger operators. arXiv:math/ 9810063v1 [math.SP].

2. Rappaz J., Sanchez Hubert J., Sanchez Palencia E., Vassiliev D. On spectral pollution in the finite element approximation of thin elastic 'membrane' shells. Numer. Math., 1997, vol. 75, pp. $473-500$. 
3. Davies E.B. Spectral enclosures and complex resonances for general selfadjoint operators. LMS J. Comput. Math., 1998, vol. 1, pp. 42-74.

4. Davies E.B., Plum M. Spectral pollution. arXiv:math/0302145v1., 2002.

5. Boffi D. et al. On the problem of spurious eigenvalues in the approximation of linear elliptic problems in mixed form. Math. of Comp., 1999, vol. 69, pp. 121-140.

6. Guebbai H. Generalized spectrum approximation and numerical computation of eigenvalues for schrödinger's operators. Lobachevskii Journal of Mathematics, 2013, vol. 34, pp. 45-60.

7. Ahues M., Largillier A., Limaye B.V. Spectral Computations for Bounded Operators. New York, Chapman and Hall/CRC, 2001.

8. Marletta M., Scheichl R. Eigenvalues in Spectral Gaps of Differential Operators. J. Spectr. Theory, 2012, vol. 2 (3), pp. 293-320.

9. Guebbai H., Largillier A. Spectra and Pseudospectra of Convection-Diffusion Operator In: Constanda C., Harris P. (eds.). Integral Methods in Science and Engineering. Boston, 2011.

10. Roach G.F. Green's Functions. New York, Cambridge University Press, 1982.

11. Atkinson K.E. The Numerical Solution of Integral Equations of the Second Kind. Cambridge, Cambridge University Press, 1997.

12. Trefethen L.N. Pseudospectra of Linear Operators. SIAM Review, 1997, vol. 39, pp. 383.

13. Laub A.J. Matrix Analysis for Scientists and Engineers. California, SIAM, 2005, 172 p.

14. Gohberg I., Goldberg S., Kaashoek M.A. Classes of Linear Operators. Vol. I. Basel, Springer, 1990.

15. Kato T. Perturbation Theory of Linear Operators. Berlin, Heidelberg, New York, SpringerVerlag, 1980.

Received 12 April 2018

Reviewed 21 May 2018

Accepted for press 26 June 2018

Khellaf Ammar, University 8 Mai 1945, Guelma, Algérie, Post-Graduate Student, e-mail: amarlasix@gmail.com; khellaf.ammar@univ-guelma.dz

For citation: Khellaf A. New sufficient conditions in the generalized spectrum approach to deal with spectral pollution. Vestnik Tambovskogo universiteta. Seriya: estestvennye i tekhnicheskie nauki - Tambov University Reports. Series: Natural and Technical Sciences, 2018, vol. 23, no. 124, pp. 595-604. DOI: 10.20310/1810-0198-2018-23-124-595-604 (In Engl., Abstr. in Russian). 


\title{
НОВЫЕ ДОСТАТОЧНЫЕ УСЛОВИЯ ОБОБЩЕННОГО СПЕКТРАЛЬНОГО ПОДХОДА ДЛЯ РЕШЕНИЯ СПЕКТРАЛЬНОГО ЗАГРЯЗНЕНИЯ
}

\author{
А. Хеллаф \\ Университет 8 мая 1945 \\ 24000, г. Гельма, Алжир, В.Р. 401 \\ E-mail: amarlasix@gmail.com; khellaf.ammar@univ-guelma.dz
}

\begin{abstract}
Аннотация. В этой работе мы предлагаем новые достаточные условия для решения задачи спектрального загрязнения с использованием метода обобщенного спектра. Мы приводим теоретическую основу обобщенного спектрального подхода, а также иллюстрируем его эффективность на численных результатах.

Ключевые слова: обобщенный спектр; оператор Шрёдингера; аппроксимация собственных значений
\end{abstract}

\section{СПИСОК ЛИТЕРАТУРЫ}

1. Aslanyan A., Davies E.B. Spectral instability for some Schrodinger operators. arXiv:math/ 9810063v1 [math.SP].

2. Rappaz J., Sanchez Hubert J., Sanchez Palencia E., Vassiliev D. On spectral pollution in the finite element approximation of thin elastic 'membrane' shells // Numer. Math. 1997. vol. 75. P. 473-500.

3. Davies E.B. Spectral enclosures and complex resonances for general selfadjoint operators // LMS J. Comput. Math. 1998. Vol. 1. P. 42-74.

4. Davies E.B., Plum M. Spectral pollution. arXiv:math/0302145v1. 2002.

5. Boffi D. et al. On the problem of spurious eigenvalues in the approximation of linear elliptic problems in mixed form // Math. of Comp. 1999. Vol. 69. P. 121-140.

6. Guebbai H. Generalized spectrum approximation and numerical computation of eigenvalues for schrödinger's operators // Lobachevskii Journal of Mathematics. 2013. Vol. 34. P. 45-60.

7. Ahues M., Largillier A., Limaye B.V. Spectral Computations for Bounded Operators. N. Y.: Chapman and Hall/CRC, 2001.

8. Marletta M., Scheichl R. Eigenvalues in Spectral Gaps of Differential Operators // J. Spectr. Theory. 2012. Vol. 2 (3). P. 293-320.

9. Guebbai H., Largillier A. Spectra and Pseudospectra of Convection-Diffusion Operator// Integral Methods in Science and Engineering. Boston, 2011.

10. Roach G.F. Green's Functions. N. Y.: Cambridge University Press, 1982.

11. Atkinson K.E. The Numerical Solution of Integral Equations of the Second Kind. Cambridge, Cambridge University Press, 1997.

12. Trefethen L.N. Pseudospectra of Linear Operators // SIAM Review. 1997. Vol. 39. P. 383. 
13. Laub A.J. Matrix Analysis for Scientists and Engineers. California: SIAM, 2005. 172 p.

14. Gohberg I., Goldberg S., Kaashoek M.A. Classes of Linear Operators. Vol. I. Basel: Springer, 1990.

15. Kato T. Perturbation Theory of Linear Operators. Second edition. Berlin; Heidelberg; New York: Springer-Verlag, 1980.

Поступила в редакцию 12 апреля 2018 г.

Прошла рецензирование 21 мая 2018 г.

Принята в печать 26 июня 2018 г.

Хеллаф Аммар, Университет 8 мая 1945, г. Гельма, Алжир, аспирант, кафедра математики, e-mail: amarlasix@gmail.com; khellaf.ammar@univ-guelma.dz

Для цитирования: Хеллаф А. Новые достаточные условия обобщенного спектрального подхода для решения спектрального загрязнения // Вестник Тамбовского университета. Серия: естественные и технические науки. Тамбов, 2018. T. 23. № 124. C. 595-604. DOI: 10.20310/1810-0198-2018-23-124-595-604 